\title{
Repeatability of methylation measures using a QIAseq targeted methyl panel and comparison with the Illumina HumanMethylation450 assay
}

\author{
Chenglong Yu' ${ }^{1}$, Pierre-Antoine Dugué ${ }^{1,2,3}$, James G. Dowty ${ }^{3}$, Fleur Hammet ${ }^{1}$, JiHoon E. Joo ${ }^{4}$, Ee Ming Wong ${ }^{1}$, \\ Mahnaz Hosseinpour ${ }^{1}$, Graham G. Giles ${ }^{1,2,3}$, John L. Hopper ${ }^{3}$, Tu Nguyen-Dumont ${ }^{1,4}$, Robert J. Maclnnis ${ }^{2,3}$ and \\ Melissa C. Southey ${ }^{1,24^{*}}$ (10)
}

\begin{abstract}
Objective: In previous studies using Illumina Infinium methylation arrays, we have identified DNA methylation marks associated with cancer predisposition and progression. In the present study, we have sought to find appropriate technology to both technically validate our data and expand our understanding of DNA methylation in these genomic regions. Here, we aimed to assess the repeatability of methylation measures made using QIAseq targeted methyl panel and to compare them with those obtained from the Illumina HumanMethylation450 (HM450K) assay. We included in the analysis high molecular weight DNA extracted from whole blood (WB) and DNA extracted from formalin-fixed paraffin-embedded tissues (FFPE).

Results: The repeatability of QIAseq-methylation measures was assessed at $40 \mathrm{CpGs}$, using the Intraclass Correlation Coefficient (ICC). The mean ICCS and 95\% confidence intervals (CI) were $0.72(0.62-0.81), 0.59(0.47-0.71)$ and 0.80 (0.73-0.88) for WB, FFPE and both sample types combined, respectively. For technical replicates measured using QIAseq and HM450K, the mean ICCS $(95 \%$ CI) were $0.53(0.39-0.68), 0.43(0.31-0.56)$ and 0.70 (0.59-0.80), respectively. Bland-Altman plots indicated good agreement between QIAseq and HM450K measurements. These results demonstrate that the QIAseq targeted methyl panel produces reliable and reproducible methylation measurements across the $40 \mathrm{CpGs}$ that were examined.
\end{abstract}

Keywords: DNA methylation, Whole blood, Formalin-fixed paraffin-embedded, Illumina Infinium methylation arrays, Targeted bisulphite sequencing, Intraclass correlation coefficient, Bland-Altman plot

\section{Introduction}

Methylation arrays, including the Illumina Infinium HumanMethylation450 (HM450K) [1] and MethylationEPIC (EPIC) [2] arrays, have enabled genome-wide measurement of DNA methylation in a number of contexts [3-10]. The application of these arrays has included large discovery initiatives in studies with large sample

\footnotetext{
*Correspondence: melissa.southey@monash.edu

${ }^{1}$ Precision Medicine, School of Clinical Sciences at Monash Health,

Monash University, Victoria, Australia

Full list of author information is available at the end of the article
}

sizes, which has been possible thanks to compatibility with clinical samples, relatively low DNA input and the cost efficiency of the array platforms [2, 11]. However, beyond the hypothesis generating initiatives, it is common for researchers to pursue findings on alternative platforms [12-16]. These platforms often need to enable a researcher-defined genomic target, for exploration of DNA methylation in specific genomic regions, not limited by CpGs included in the array designs. Such alternative platforms often also need to support an expanded sample size to adequately validate findings from initial array-based discovery-focused studies. 
Here we used a custom QIAseq targeted methyl panel (Qiagen, Hilden, Germany) to measure DNA methylation in DNA samples extracted from whole blood (WB) and formalin-fixed paraffin-embedded (FFPE) tissues. The objectives of this study were to evaluate the repeatability of methylation measures by comparing the same technology duplicates (QIAseq) and different technology replicates (QIAseq and HM450K) based on the same DNA samples. The results will provide information for designing and conducting future larger studies aimed at targeted DNA methylation characterization.

\section{Main text}

\section{Materials and methods}

\section{Sample selection}

The DNA samples included in this study were selected from participants in the Australian Breast Cancer Family Registry $[17,18]$. We included 26 DNA samples extracted from $\mathrm{WB}$ and 7 DNA samples extracted from FFPE breast cancer tissues. As shown in Table 1, 7 WB and 3 FFPE DNA samples were duplicated, i.e. duplicated DNA samples were included in the QIAseq targeted methyl panel sequencing, which we used to assess repeatability. Additionally, 25 of the 26 WB DNA samples and all 7 FFPE DNA samples had DNA methylation measured using the HM450K assay, as described previously [1821 ], thus these samples were used to assess repeatability when using either of QIAseq or HM450K. All duplicated samples were from the same DNA extraction.

\section{QIAseq targeted methyl panel library preparation and sequencing}

The QIAseq targeted methyl panel was designed by the manufacturer (Qiagen, Hilden, Germany), to target 41 CpGs (in 28 genomic regions with total breadth of $8,673 \mathrm{bp}$, see Additional file 1: Table S1), identified in our previous studies of heritable methylation marks associated with cancer risk using the EPIC array [22]. Briefly, 100 ng WB and 200 ng FFPE DNA was bisulphite converted according to the manufacturer's instructions with the EPITECT Fast Bisulfite Sequencing (BS) conversion kit. Bisulphite-converted DNA was used as the input template to create targeted libraries as per the QIAseq targeted methyl panel protocol. Enriched targeted sample libraries were amplified for 19 or 21 (genomic DNA or FFPE DNA) cycles respectively. Samples quantified by analysis on a D1000 HS ScreenTape (Agilent, Santa Clara, CA, USA) were pooled in equimolar concentrations and $10 \mathrm{pM}$ pooled library was loaded for sequencing using 500 Cycle v2 MiSeq reagent kit (Illumina, San Diego, CA, USA).

Paired-end reads were mapped to the human genome reference (hg19) and methylation levels were called using the default settings of CLC Genomics Workbench (Qiagen). At a given CpG site, the DNA methylation level, a beta value between 0 (unmethylated) and 1 (fully methylated), was calculated as methylated coverage (the number of reads with evidence of methylation in this position) divided by context coverage (the number of reads conforming to the selected methylation context), see [23] for details. We assigned methylation values to "missing" when the context coverage was less than $30 \mathrm{X}$.

\section{Illumina Infinium HM450K array}

HM450K BeadChip (Illumina, San Diego, CA, USA) DNA methylation measurements were available for 25/26 WB and 7/7 FFPE DNA samples assessed by QIAseq, and described previously [18-21]. Of the 41 CpGs of interest, which were previously identified as heritable cancer-associated marks using the EPIC array, only 24 were present on the HM450K array (Table 1) and therefore had available data for the tested samples. Two of these 24 CpGs (Illumina cg02722613 and cg07158503) did not pass quality control (QC) for the FFPE DNA samples (measures with a probe detection P-value of $<0.05$ were assigned to "missing" and CpGs with $>20 \%$ missing values across samples were excluded in the original study [18]) and were excluded from further analysis (Table 1). Beta values obtained using the HM450K array data were used for comparison analysis with the QIAseq panel.

\section{Data analysis}

The distributions of paired beta values were represented graphically for repeated measures at each $\mathrm{CpG}$, i.e. duplicated measures by the QIAseq panel, or replicated measures by QIAseq and HM450K platforms, which were assessed from the same DNA samples. We also used boxplots to show median and interquartile range (IQR) of beta values of QIAseq and HM450K platforms across different DNA sample types (WB, FFPE and sample types combined). Methylation values obtained for the two platforms were further compared using a Wilcoxon signedrank test because the distributions of overall methylation values for the two platforms and methylation differences between the two platforms were not Gaussian (see "Results" section).

For each $\mathrm{CpG}$, we estimated the repeatability of measurements by calculating the intraclass correlation coefficient (ICC) under a mixed effects model framework. The ICC is calculated as the variance within groups (i.e. duplicated DNA samples) means $V_{G}$ divided by the sum of variances of the group-level and random error (residual) specific to each measure $V_{R}: I C C=V_{G} /\left(V_{G}+V_{R}\right)$. The ICC was estimated using the $\mathrm{R}$ package $r p t R$ [24] with a confidence interval $(\mathrm{CI})$ quantified via parametric 
Table 1 Forty-one CpGs and their intraclass correlation coefficients (ICC) using 1) duplicated samples both measured using Qiagen targeted methyl panel, 2) replicated samples measured using Qiagen targeted methylated panel and Illumina HM450K assay

\begin{tabular}{|c|c|c|c|c|c|c|c|c|}
\hline \multicolumn{3}{|c|}{$\begin{array}{l}\text { CpGs included on the QIAseq } \\
\text { targeted methyl panel (and Illumina } \\
\text { EPIC assay) }\end{array}$} & \multicolumn{3}{|c|}{$\begin{array}{l}\text { Repeatability }(\mathrm{ICC}, 95 \% \mathrm{Cl}) \text {, using duplicated } \\
\text { samples both measured with Qiagen targeted } \\
\text { methyl panel }\end{array}$} & \multicolumn{3}{|c|}{$\begin{array}{l}\text { Repeatability (ICC, } 95 \% \mathrm{Cl}) \text {, using replicated samples } \\
\text { measured with Qiagen targeted methyl panel and } \\
\text { Illumina HM450K assay }\end{array}$} \\
\hline $\mathrm{CpG}^{\wedge}$ & Chr & Position* & $\begin{array}{l}\text { WB samples } \\
7 \text { pairs }\end{array}$ & $\begin{array}{l}\text { FFPE samples } \\
3 \text { pairs }\end{array}$ & $\begin{array}{l}\text { Combined } \\
\text { samples } 10 \\
\text { pairs }\end{array}$ & $\begin{array}{l}\text { WB samples } \\
25 \text { pairs }\end{array}$ & $\begin{array}{l}\text { FFPE samples } \\
7 \text { pairs }\end{array}$ & $\begin{array}{l}\text { Combined samples } \\
32 \text { pairs }\end{array}$ \\
\hline $\operatorname{cg} 18072778^{*}$ & 1 & $148,203,924$ & $0.54(0-0.90)$ & $0.37(0-0.94)$ & $0.85(0.54-0.96)$ & $0(0-0.39)$ & $0.4(0-0.88)$ & $0.76(0.56-0.88)$ \\
\hline cg04546999* & 1 & $152,956,430$ & $0.19(0-0.75)$ & $0.80(0-0.99)$ & $0.92(0.74-0.98)$ & $0.09(0-0.47)$ & $0(0-0.68)$ & $0.8(0.63-0.9)$ \\
\hline cg17714793 & 1 & $153,538,431$ & $0(0-0.63)$ & $0.99(0.47-1)$ & $0.72(0.24-0.92)$ & & & \\
\hline cg01608070 & 1 & $157,853,274$ & $0.98(0.89-1)$ & $0.46(0-0.96)$ & $0.96(0.84-0.99)$ & & & \\
\hline cg21501207 & 1 & $162,383,000$ & $0.89(0.56-0.98)$ & $0.28(0-0.94)$ & $0.80(0.43-0.94)$ & & & \\
\hline cg26237810 & 1 & $200,669,215$ & $0.52(0-0.89)$ & $0.96(0-1)$ & $0.78(0.29-0.95)$ & & & \\
\hline $\operatorname{cg} 26354017^{*}$ & 1 & $205,819,088$ & $0.92(0.64-0.98)$ & $0.70(0-0.97)$ & $0.94(0.78-0.98)$ & $0.83(0.67-0.92)$ & $0.46(0-0.86)$ & $0.83(0.69-0.92)$ \\
\hline $\operatorname{cg} 14159672^{*}$ & 1 & $205,819,179$ & $0.95(0.71-0.99)$ & $0.96(0.27-1)$ & $0.98(0.94-1.00)$ & $0.79(0.57-0.9)$ & $0.76(0.16-0.95)$ & $0.82(0.66-0.91)$ \\
\hline $\operatorname{cg} 14893161^{*}$ & 1 & $205,819,252$ & $0.92(0.63-0.98)$ & $0.99(0.59-1)$ & $0.97(0.89-0.99)$ & $0.94(0.86-0.97)$ & $0.73(0-0.94)$ & $0.93(0.86-0.96)$ \\
\hline cg05841700 & 1 & $205,819,384$ & $0.96(0.80-0.99)$ & $0.68(0-0.98)$ & $0.96(0.88-0.99)$ & & & \\
\hline $\operatorname{cg} 24503407^{*}$ & 1 & $205,819,493$ & $0.92(0.65-0.98)$ & $0(0-0.96)$ & $0.95(0.82-0.99)$ & $0.87(0.74-0.94)$ & $0.8(0.02-0.98)$ & $0.88(0.76-0.94)$ \\
\hline cg16334093 & 1 & $205,819,601$ & $0.87(0.43-0.97)$ & $0.64(0-0.97)$ & $0.87(0.54-0.97)$ & & & \\
\hline cg07157834* & 1 & $205,819,610$ & $0.90(0.47-0.98)$ & $0(0-0.89)$ & $0.94(0.75-0.98)$ & $0.6(0.29-0.81)$ & $0.26(0-0.82)$ & $0.65(0.39-0.81)$ \\
\hline cg20004147 & 2 & $65,718,931$ & $0.98(0.92-1)$ & $0.98(0.47-1)$ & $0.98(0.94-1.00)$ & & & \\
\hline cg21824770 & 2 & $243,012,164$ & $0.28(0-0.78)$ & $0(0-0.95)$ & $0.88(0.58-0.97)$ & & & \\
\hline cg10123377 & 3 & $42,387,525$ & $0.97(0.87-0.99)$ & $0.68(0-0.97)$ & $0.92(0.72-0.98)$ & & & \\
\hline cg01760119 & 3 & $101,661,383$ & $0.12(0-0.7)$ & $0.87(0-0.99)$ & $0.98(0.93-1.00)$ & & & \\
\hline $\operatorname{cg} 12012426^{\dagger}$ & 4 & $1,366,464$ & - & - & - & - & - & - \\
\hline cg19704288* & 4 & $1,582,182$ & $0.24(0-0.79)$ & $0.76(0-0.98)$ & $0.81(0.43-0.95)$ & $0(0-0.38)$ & $0.81(0.33-0.96)$ & $0.82(0.66-0.91)$ \\
\hline cg02722613, \#\# & 4 & $25,162,899$ & $0.75(0.18-0.94)$ & $0(0-0.89)$ & $0.77(0.38-0.93)$ & $0.87(0.71-0.94)$ & - & - \\
\hline cg19182683 & 4 & $183,730,519$ & $0.98(0.91-1)$ & $0.94(0-1)$ & $0.97(0.89-0.99)$ & & & \\
\hline cg07158503* \# & 5 & $135,415,693$ & $0.63(0-0.92)$ & $0.96(0.12-1)$ & $0.79(0.35-0.94)$ & $0.55(0.22-0.77)$ & - & - \\
\hline $\operatorname{cg} 11608150^{*}$ & 5 & $135,415,949$ & $0.71(0.02-0.93)$ & $0.17(0-0.97)$ & $0.61(0.03-0.89)$ & $0.91(0.81-0.96)$ & $0.44(0-0.94)$ & $0.84(0.7-0.92)$ \\
\hline cg06478886* & 5 & $135,416,030$ & $0.79(0.24-0.96)$ & $0.98(0.44-1)$ & $0.85(0.54-0.96)$ & $0.94(0.86-0.97)$ & $0.31(0-0.83)$ & $0.9(0.8-0.95)$ \\
\hline cg04481923* & 5 & $135,416,206$ & $0.74(0.05-0.95)$ & $0(0-0.89)$ & $0.23(0-0.71)$ & $0.59(0.28-0.78)$ & $0.74(0.04-0.95)$ & $0.62(0.34-0.79)$ \\
\hline cg06536614* & 5 & $135,416,381$ & $0.81(0.24-0.95)$ & $0.47(0-0.95)$ & $0.63(0.08-0.88)$ & $0.71(0.46-0.86)$ & $0(0-0.68)$ & $0.7(0.47-0.84)$ \\
\hline cg25340688* & 5 & $135,416,398$ & $0.79(0.21-0.96)$ & $0.99(0.66-1)$ & $0.85(0.47-0.95)$ & $0.65(0.36-0.83)$ & $0.50(0-0.88)$ & $0.7(0.47-0.84)$ \\
\hline $\operatorname{cg} 26896946^{*}$ & 5 & $135,416,405$ & $0.82(0.29-0.97)$ & $0.55(0-0.96)$ & $0.76(0.29-0.93)$ & $0.68(0.4-0.84)$ & $0.31(0-0.81)$ & $0.72(0.53-0.85)$ \\
\hline cg00124993* & 5 & $135,416,412$ & $0.82(0.21-0.96)$ & $0.96(0.13-1)$ & $0.87(0.55-0.97)$ & $0.71(0.45-0.86)$ & $0.64(0-0.92)$ & $0.73(0.52-0.86)$ \\
\hline $\operatorname{cg} 18797653^{*}$ & 5 & $135,416,613$ & $0.89(0.54-0.97)$ & $0(0-0.87)$ & $0.89(0.59-0.97)$ & $0.50(0.14-0.74)$ & $0.76(0.14-0.95)$ & $0.56(0.25-0.75)$ \\
\hline cg09483595* & 5 & $158,878,381$ & $0.98(0.91-1)$ & $0.71(0-0.98)$ & $0.92(0.68-0.98)$ & $0(0-0.4)$ & $0.61(0-0.91)$ & $0.94(0.88-0.97)$ \\
\hline cg13373914 & 7 & $67,323,067$ & $0.12(0-0.74)$ & $1.00(0.94-1)$ & $0.90(0.63-0.98)$ & & & \\
\hline cg05141217 & 8 & $28,491,379$ & $0.96(0.80-0.99)$ & $1.00(0.9-1)$ & $0.97(0.87-0.99)$ & & & \\
\hline cg26708920 & 10 & $13,826,318$ & $0.94(0.72-0.99)$ & $0.96(0.01-1)$ & $0.96(0.86-0.99)$ & & & \\
\hline $\operatorname{cg} 20054939^{*}$ & 12 & $133,614,314$ & $0.66(0-0.92)$ & $0(0-0)$ & $0.08(0-0.63)$ & $0(0-0.41)$ & $0(0-0.72)$ & $0(0-0.36)$ \\
\hline cg20124410 & 13 & $107,333,224$ & $0.58(0-0.9)$ & $0(0-0.87)$ & $0.32(0-0.73)$ & & & \\
\hline cg10829391 & 14 & $101,069,717$ & $0.95(0.75-0.99)$ & $0.59(0-0.97)$ & $0.87(0.57-0.97)$ & & & \\
\hline $\operatorname{cg} 26748794^{*}$ & 16 & $88,804,052$ & $0.97(0.84-0.99)$ & $0.59(0-0.97)$ & $0.89(0.63-0.97)$ & $0.67(0.41-0.83)$ & $0.69(0.01-0.92)$ & $0.67(0.41-0.83)$ \\
\hline cg20443278* & 17 & $77,962,099$ & $0.62(0-0.91)$ & $0.78(0-0.99)$ & $0.96(0.85-0.99)$ & $0(0-0.38)$ & $0.24(0-0.8)$ & $0.83(0.67-0.91)$ \\
\hline $\operatorname{cg} 14150973^{*}$ & 19 & $40,950,432$ & $0(0-0.67)$ & $0(0-0.88)$ & $0(0-0.54)$ & $0(0-0.39)$ & $0(0-0.65)$ & $0(0-0.35)$ \\
\hline $\operatorname{cg} 17884856^{*}$ & 20 & $44,334,913$ & $0.93(0.69-0.99)$ & $0.84(0-0.99)$ & $0.88(0.59-0.97)$ & $0.89(0.79-0.95)$ & $0.05(0-0.68)$ & $0.59(0.32-0.78)$ \\
\hline
\end{tabular}

\footnotetext{
${ }^{\wedge} \mathrm{CpG}$ names used Illumina nomenclature

" CpGs included on the Infinium HM450K BeadChip. 24 and 22 CpGs were detected and passed QC on HM450K platform for WB and FFPE samples, respectively

* Genomic coordinates are based on human genome assembly hg19

\# CpGs that did not pass QC on HM450K platform for the FFPE DNA samples

${ }^{\dagger}$ CpGs that did not pass QC on the QIAseq targeted methyl panel
} 
bootstrapping (1000 times). The ICC ranges from 0 to 1 , and the higher the ICC, the higher the similarity between replicated values.

We also generated Bland-Altman plots to evaluate the agreement between repeated measures for each $\mathrm{CpG}$, and used the $95 \%$ limits of agreement for each comparison (average difference \pm 1.96 standard deviation (SD) of the difference). As the sample size for FFPE was small, we only examined Bland-Altman plots for WB and combined (WB and FFPE) DNA samples.

\section{Results}

Average coverage of the targeted regions (Additional file 1: Table S1) sequenced using the QIAseq targeted methyl panel for the 26 WB, 7 FFPE and 33 samples combined was $447 \times($ range $190 \times-1268 \times), 258 \times($ range $66 \times-539 \times$ ) and 408X (range $66 \times-1268 \times$ ) with $92.4 \%$, $90.4 \%$ and $92.0 \%$ reads mapped, respectively. Average context coverage of each of the $41 \mathrm{CpGs}$ for WB, FFPE and combined DNA samples was also shown in Additional file 1: Table S2. One CpG at chr4:1,366,464 (Illumina cg12012426) was excluded from the analysis due to poor coverage ( $>20 \%$ samples had less than $30 \times$ coverage), the remaining $40 \mathrm{CpGs}$ across 15 chromosomes were retained for the analysis (Table 1).

The distributions of paired beta values for duplicated samples measured using the QIAseq panel (40 CpGs) and replicated samples measured using the QIAseq and HM450K platforms (24 CpGs) are shown in Additional file 1: Figures S1 and S2, respectively, showing good consistency of repeated measures.

Table 1 shows the repeatability (ICC) of the QIAseq panel measurements for all 40 CpGs. The mean (95\% CI) ICC across 40 CpGs was $0.72(0.62-0.81)$ for WB samples, 0.59 (0.47-0.71) for FFPE samples, and 0.80 (0.730.88 ) for the two sample types combined. The majority of CpGs, 33/40 and 36/40, had an ICC greater than 0.5 for $\mathrm{WB}$ and all sample types combined, respectively. The estimated ICC was low for CpGs with more extreme values (closer to 0 or 1 ), for which there was low methylation variability. For instance, Illumina cg20054939 and cg14150973 had poor repeatability (ICC $<0.1$ in all samples combined) but the beta value SDs were lower than 0.02, as shown in Fig. 1 and Additional file 1: Figure S1.

Table 1 also shows the repeatability of methylation measures at $24 \mathrm{CpGs}$ when using the QIAseq and HM450K technologies. The mean (95\% CI) ICC across these 24 CpGs was $0.53(0.39-0.68)$ for WB samples, $0.43(0.31-0.56)$ for FFPE samples and $0.70(0.59-0.80)$ when sample types were combined. Similar to observations made above, the majority of CpGs, $17 / 24$ and 20/22, had an ICC greater than 0.5 for WB and all sample types combined, respectively, and those CpGs that had low
ICC predominantly showed little variability (Fig. 1 and Additional file 1: Figure S2).

Additional file 1: Figure S3 presents boxplots of beta values at each of the $24 \mathrm{CpGs}$ for QIAseq and HM450K technology across sample types. We also show in Fig. 2 boxplots of methylation value distributions for all $\mathrm{CpGs}$ combined. Additional file 1: Figure S4 shows that the distributions of overall methylation values for the two platforms and methylation differences between the two platforms. As the distributions of methylation differences between the two platforms were not Gaussian, a Wilcoxon signed-rank test was used to compare them. On average, the QIAseq targeted panel produced lower methylation levels than those obtained using the HM450K assay; WB: median [IQR] for QIAseq: 0.36 [0.04-0.67] and HM450K: 0.53 [0.13-0.68], Wilcoxon signed-rank test, $\mathrm{P}<2 \times 10^{-16}$; FFPE: median [IQR] for QIAseq: 0.52 [0.19-0.72] and for HM450K: 0.58 [0.320.71 ], $\mathrm{P}=0.01$; all samples combined: median [IQR] for QIAseq: 0.37 [0.06-0.69] and HM450K: 0.54 [0.17-0.70], $\mathrm{P}<2 \times 10^{-16}$

The agreement of repeated measures using the QIAseq and HM450K platforms (24 CpGs) was also assessed using Bland-Altman plots as shown in Additional file 1: Figures S5 (WB) and S6 (sample types pooled), respectively. There was overall good agreement of the QIAseq and HM450K measures. For WB DNA, the mean of differences of the two measures was within the $95 \%$ limits of agreement at 21 of the 24 CpGs, all 25 samples were within the $95 \%$ limits of agreement at 10 of the $21 \mathrm{CpGs}$, and a maximum of 2 samples were beyond at the remaining 11 CpGs (Additional file 1: Figure S5). When sample types (WB and FFPE) were combined, the mean of differences of the two measures was within the $95 \%$ limits of agreement at all the $22 \mathrm{CpGs}$, all 32 samples were within the $95 \%$ limits of agreement at 4 of the $22 \mathrm{CpGs}$, and a maximum of 4 samples were beyond at the remaining 18 CpGs (Additional file 1: Figure S6). The figures showed that most outliers in the combined data were FFPE DNAs.

\section{Discussion}

Overall, our results demonstrate that the QIAseq targeted methyl panel sequencing can produce reliable and repeatable methylation measures. Our analyses included ICCs and Bland-Altman plots, both showing that the QIAseq and HM450K platforms have overall good repeatability and agreement, especially for highmolecular weight WB DNA samples; this was despite the somewhat higher methylation values produced using the HM450K assay, particularly at lower ranges. The low ICCs observed at some CpGs, e.g. 7/40 had an ICC less than 0.5 for duplicated WB samples of QIAseq 


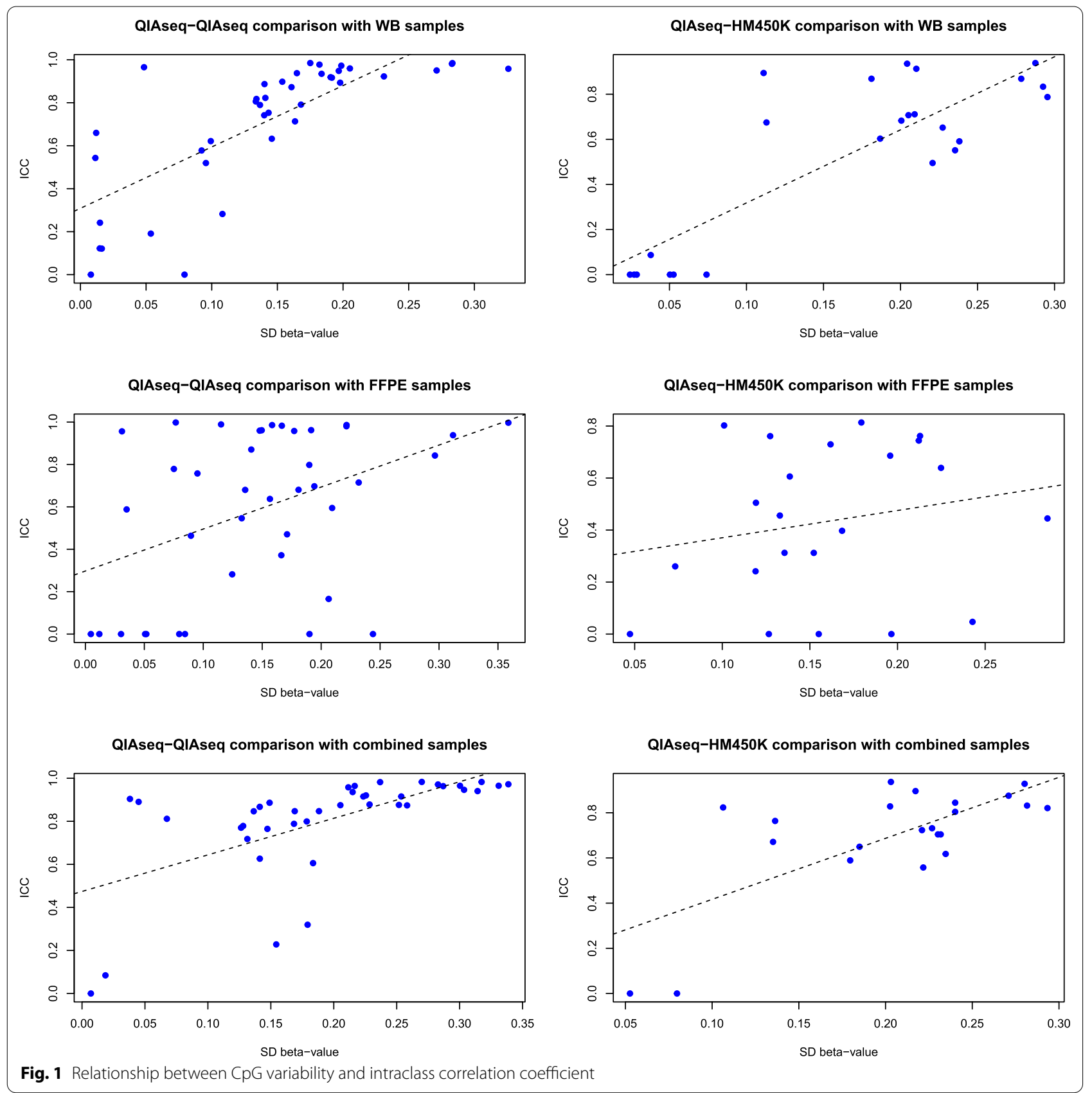

measurements, were essentially due to the low methylation variability observed at these CpGs (typically an SD across samples lower than 5\% methylation), making it difficult to distinguish measures between samples, rather than poor measurement quality. Several factors may explain small differences in methylation levels between duplicates, including biological (e.g. average across a collection of cells) and technical (e.g. batch effects), so that the observed repeatability is necessarily imperfect, both within and across measurement technology.
QIAseq targeted methyl panel sequencing can not only reliably reproduce the Infinium array data but also enable the characterisation of DNA methylation in the surrounding genomic region. Although not presented here, the QIAseq technology provides information about the DNA methylation status of the 28 targeted regions, facilitating future extended analyses of the regions around the CpGs that we have identified to be associated with cancer riskbeyond the capacity of the genome-wide array technology. This platform can therefore support an expanded sample 


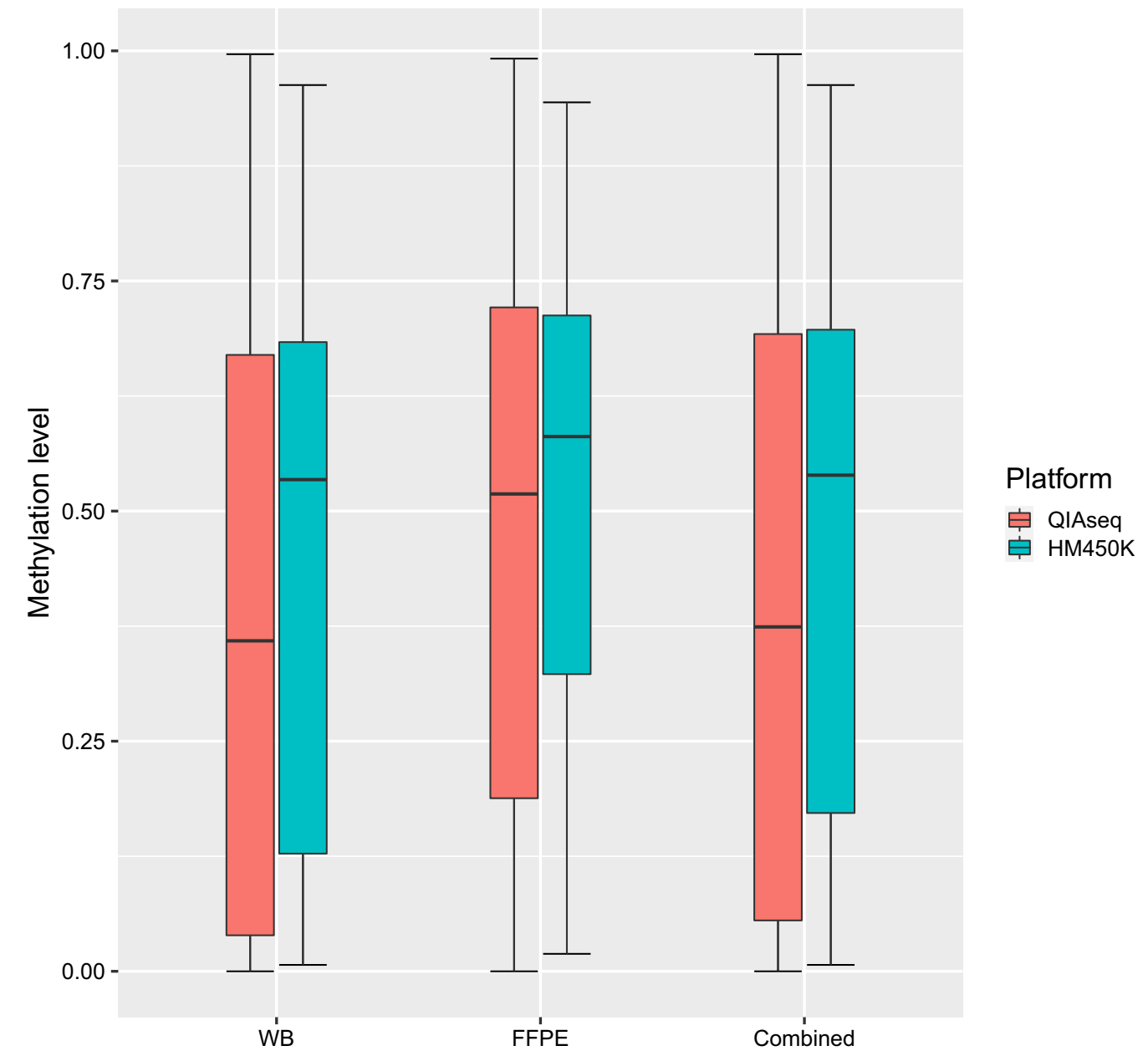

Fig. 2 Overall methylation measures (24 CpGs) using Qiaseq targeted methyl panel or Illumina HM450K assay

size to adequately validate findings from initial arraybased discovery-focused studies and support further genomic region-focused exploration of DNA methylation.

\section{Limitations}

Our study has three main limitations. First, only a limited number of CpGs were included in this analysis. These CpGs were of particular interest to us and may not be representative of all CpGs across the genome, however the included CpGs were spread across several chromosomes and genomic regions. Second, our sample sizes were small, especially for FFPE DNA samples and thus our findings would require further confirmation using more duplicate pairs. Nevertheless, our aim was not to obtain very precise estimation of ICCs but rather to confirm that these were good for most CpGs. Third, there was insufficient coverage $(<30 \times)$ for some targeted CpGs in some samples, which could be addressed by increasing sequencing depth.

\section{Abbreviations}

CpG: 5'-C-phosphate-G-3'; WB: Whole blood; FFPE: Formalin-fixed paraffinembedded tissue; ICC: Intraclass correlation coefficient; HM450K: HumanMethylation450; EPIC: MethylationEPIC; BS: Bisulfite sequencing; QC: Quality control; IQR: Interquartile range; Cl: Confidence interval; SD: Standard deviation.

\section{Supplementary Information}

The online version contains supplementary material available at https://doi. org/10.1186/s13104-021-05809-z.

Additional file 1: Supplementary Tables and Figures.

\section{Acknowledgements}

We thank all the participants in this study, the entire team of the Australian Breast Cancer Family registry (BCFR-AU) and past and current investigators.

\section{Authors' contributions}

Conceptualization, MCS; methodology, MCS, P-AD, and CY; formal analysis, CY, FH and P-AD; resources, MCS, JLH, GGG, RJM, and JLH; writing original draft MCS, P-AD, CY and FH; data generation, FH, EMW, JEJ; writing reviewing and editing, JLH, GGG, MH, TN-D, EMW, RJM, JEJ, and JGD; project administration, 
funding acquisition, MCS, RJM, P-AD and JLH. All authors read and approved the final manuscript.

\section{Funding}

This work was funded by the U.S. National Institute of Health (Grant Number RO1CA159868). The ABCFR was supported in Australia by the National Health and Medical Research Council, the New South Wales Cancer Council, the Victorian Health Promotion Foundation, the Victorian Breast Cancer Research Consortium, Cancer Australia, and the National Breast Cancer Foundation. The six sites of the Breast Cancer Family Registry (BCFR) were supported by grant UM1 CA164920 from the U.S. National Cancer Institute. The content of this manuscript does not necessarily reflect the views or policies of the National Cancer Institute or any of the collaborating centers in the BCFR, nor does mention of trade names, commercial products, or organizations imply endorsement by the U.S. Government or the BCFR. TN-D is a National Breast Cancer Foundation (Australia) Career Development Fellow (ECF-17-001). MCS is a National Health and Medical Research Council (NMHRC, Australia) Senior Research Fellow (GNT1155163). This work was supported by an NHMRC Program grant (GNT1074383), and Monash University, Melbourne, Australia.

\section{Availability of data and materials}

The datasets used and/or analysed during the current study are available from the corresponding author on reasonable request.

\section{Declarations}

\section{Ethics approval and consent to participate}

Written informed consent was obtained from all individual participants included in the study. This study was approved by the Human Research Ethics Committees of the University of Melbourne (\# 1441420.1) and Monash University (21926).

\section{Consent for publication}

Not applicable.

\section{Competing interests}

The authors have no competing interests.

\section{Author details}

'Precision Medicine, School of Clinical Sciences at Monash Health, Monash University, Victoria, Australia. ${ }^{2}$ Cancer Council Victoria, Cancer Epidemiology Division, Melbourne, Australia. ${ }^{3}$ Centre for Epidemiology and Biostatistics, Melbourne School of Population and Global Health, The University of Melbourne, Parkville, VIC, Australia. ${ }^{4}$ Department of Clinical Pathology, The Melbourne Medical School, The University of Melbourne, Melbourne, VIC, Australia.

\section{Received: 31 August 2021 Accepted: 11 October 2021}

Published online: 24 October 2021

\section{References}

1. Bibikova M, Barnes B, Tsan C, Ho V, Klotzle B, Le JM, et al. High density DNA methylation array with single $\mathrm{CpG}$ site resolution. Genomics. 2011;98(4):288-95.

2. Pidsley R, Zotenko E, Peters TJ, Lawrence MG, Risbridger GP, Molloy $P$, et al. Critical evaluation of the Illumina MethylationEPIC BeadChip microarray for whole-genome DNA methylation profiling. Genome Biol. 2016;17(1):208

3. Dugué PA, Brinkman MT, Milne RL, Wong EM, FitzGerald LM, Bassett JK, et al. Genome-wide measures of DNA methylation in peripheral blood and the risk of urothelial cell carcinoma: a prospective nested case-control study. Br J Cancer. 2016;115(6):664-73.

4. Baglietto L, Ponzi E, Haycock P, Hodge A, Bianca Assumma M, Jung CH, et al. DNA methylation changes measured in pre-diagnostic peripheral blood samples are associated with smoking and lung cancer risk. Int J Cancer. 2017;140(1):50-61.

5. Dugué PA, Bassett JK, Joo JE, Jung CH, Ming Wong E, Moreno-Betancur $M$, et al. DNA methylation-based biological aging and cancer risk and survival: Pooled analysis of seven prospective studies. Int J Cancer. 2018;142(8):1611-9.

6. Dugué PA, Dowty JG, Joo JE, Wong EM, Makalic E, Schmidt DF, et al. Heritable methylation marks associated with breast and prostate cancer risk. Prostate. 2018;78(13):962-9.

7. Bodelon C, Ambatipudi S, Dugué PA, Johansson A, Sampson JN, Hicks B, et al. Blood DNA methylation and breast cancer risk: a meta-analysis of four prospective cohort studies. Breast Cancer Res. 2019;21(1):62.

8. Wong EM, Southey MC, Terry MB. Integrating DNA methylation measures to improve clinical risk assessment: are we there yet? The case of BRCA1 methylation marks to improve clinical risk assessment of breast cancer. $\mathrm{Br}$ J Cancer. 2020;122(8):1133-40.

9. Suman M, Dugué PA, Wong EM, Joo JE, Hopper JL, Nguyen-Dumont T, et al. Association of variably methylated tumour DNA regions with overall survival for invasive lobular breast cancer. Clin Epigenetics. 2021;13(1):11.

10. Dugué PA, Yu C, McKay T, Wong EM, Joo JE, Tsimiklis H, et al. VTRNA2-1: genetic variation, heritable methylation and disease association. Int J Mol Sci. 2021;22(5):2535.

11. Roessler J, Ammerpohl O, Gutwein J, Hasemeier B, Anwar SL, Kreipe H, et al. Quantitative cross-validation and content analysis of the 450k DNA methylation array from Illumina. Inc BMC Res Notes. 2012;5(1):210.

12. Teh $A L$, Pan $H$, Lin $X$, Lim $Y l$, Patro CP, Cheong CY, et al. Comparison of methyl-capture sequencing vs. Infinium 450K methylation array for methylome analysis in clinical samples. Epigenetics. 2016;11(1):36-48.

13. Šestáková Š, Šálek C, Remešová H. DNA methylation validation methods: a coherent review with practical comparison. Biological procedures online. 2019;21(1):19.

14. Shu C, Zhang X, Aouizerat BE, Xu K. Comparison of methylation capture sequencing and Infinium MethylationEPIC array in peripheral blood mononuclear cells. Epigenetics Chromatin. 2020;13(1):51.

15. De Chiara L, Leiro-Fernandez V, Rodríguez-Girondo M, Valverde D, BotanaRial MI, Fernández-Villar A. Comparison of bisulfite pyrosequencing and methylation-specific qPCR for Methylation Assessment. Int J Mol Sci. 2020;21(23):9242.

16. Lin N, Liu J, Castle J, Wan J, Shendre A, Liu Y, et al. Genome-wide DNA methylation profiling in human breast tissue by illumina TruSeq methyl capture EPIC sequencing and Infinium MethylationEPIC BeadChip microarray. Epigenetics. 2021;16(7):754-69.

17. John EM, Hopper JL, Beck JC, Knight JA, Neuhausen SL, Senie RT, et al. The Breast Cancer Family Registry: an infrastructure for cooperative multinational, interdisciplinary and translational studies of the genetic epidemiology of breast cancer. Breast Cancer Res. 2004;6(4):R375-89.

18. Scott CM, Wong EM, Joo JE, Dugué PA, Jung $\mathrm{CH}, \mathrm{O}^{\prime}$ Callaghan $\mathrm{N}$, et al. Genome-wide DNA methylation assessment of 'BRCA1-like'early-onset breast cancer: Data from the Australian Breast Cancer Family Registry. Exp Mol Pathol. 2018:105(3):404-10.

19. Wong EM, Joo JE, McLean CA, Baglietto L, English DR, Severi G, et al. Tools for translational epigenetic studies involving formalin-fixed paraffin-embedded human tissue: applying the Infinium HumanMethyation450 BeadChip assay to large population-based studies. BMC Res Notes. 2015;8(1):543.

20. Joo JE, Dowty JG, Milne RL, Wong EM, Dugué PA, English D, et al. Heritable DNA methylation marks associated with susceptibility to breast cancer Nat Commun. 2018:9(1):867.

21. Dugué PA, English DR, Maclnnis RJ, Jung CH, Bassett JK, FitzGerald LM, et al. Reliability of DNA methylation measures from dried blood spots and mononuclear cells using the HumanMethylation450k BeadArray. Sci Rep. 2016;6(1):30317.

22. Dowty JG, Yu C, Hosseinpour M, Joo JE, Wong EM, Nguyen-Dumont T, et al. Heritable methylation marks associated with prostate cancer risk. Submitted. 2021

23. https://resources.qiagenbioinformatics.com/manuals/clcgenomicswork bench/current/index.php?manual=Call_Methylation_Levels.html

24. Stoffel MA, Nakagawa S, Schielzeth H. rptR: Repeatability estimation and variance decomposition by generalized linear mixed-effects models. Methods Ecol Evol. 2017;8(11):1639.

\section{Publisher's Note}

Springer Nature remains neutral with regard to jurisdictional claims in published maps and institutional affiliations. 